\title{
Proteogenomic Characterization of Novel x-Type High Molecular Weight Glutenin Subunit 1Ax1.1
}

\author{
Miguel Ribeiro ${ }^{1,2}$, Emmanuelle Bancel ${ }^{3}$, Annie Faye ${ }^{3}$, Mireille Dardevet ${ }^{3}$, Catherine Ravel $^{3}$, \\ Gérard Branlard ${ }^{3}$ and Gilberto Igrejas ${ }^{1,2, *}$
}

1 Department of Genetics and Biotechnology, University of Trás-os-Montes and Alto Douro, 5001-801 Vila Real, Portugal; E-Mail: jmribeiro@utad.pt

2 Institute for Biotechnology and Bioengineering, Centre of Genomics and Biotechnology, University of Trás-os-Montes and Alto Douro, 5001-801 Vila Real, Portugal

3 INRA UMR1095 UBP Génétique Diversité et Ecophysiologie des Céréales, 63039 Clermont-Ferrand, France; E-Mails: emmanuelle.bancel@clermont.inra.fr (E.B.); afaye@clermont.inra.fr (A.F.); mireille.dardevet@clermont.inra.fr (M.D.); catherine.ravel@clermont.inra.fr (C.R.); gerard.branlard@clermont.inra.fr (G.B.)

* Author to whom correspondence should be addressed; E-Mail: gigrejas@utad.pt; Tel.: +351-259-350-530; Fax: +351-259-350-480.

Received: 5 November 2012; in revised form: 12 February 2013 / Accepted: 25 February 2013 / Published: 11 March 2013

\begin{abstract}
Analysis of Portuguese wheat (Triticum aestivum L.) landrace 'Barbela' revealed the existence of a new x-type high molecular weight-glutenin subunit (HMW-GS) encoded at the Glu-A1 locus, which we named 1Ax1.1. Using one-dimensional and two-dimensional electrophoresis and mass spectrometry, we compared subunit 1Ax1.1 with other subunits encoded at the Glu-A1 locus. Subunit 1Ax1.1 has a theoretical molecular weight of 93,648 $\mathrm{Da}$ (or 91,508 $\mathrm{Da}$ for the mature protein) and an isoelectric point (pI) of about 5.7, making it the largest and most acidic HMW-GS known to be encoded at Glu-A1. Specific primers were designed to amplify and sequence $2601 \mathrm{bp}$ of the Glu-Al locus from the 'Barbela 28' wheat genome. A very high level of identity was found between the sequence encoding $1 \mathrm{Ax} 1.1$ and those encoding other alleles of the locus. The major difference found was an insertion of 36 amino acids in the central repetitive domain.
\end{abstract}

Keywords: Triticum aestivum L.; wheat; storage proteins; glutenins; HMW-GS; Glu-A1; 1Ax1.1; MALDI-TOF-MS 


\section{Introduction}

Wheat is one of the most widely grown crops in the world and a major source of protein in the human diet. Roughly $85 \%$ of the total protein contained within wheat endosperm is gluten, a very large complex responsible for the unique viscoelastic properties of wheat dough [1,2]. The major components of gluten are the monomeric gliadins and the polymeric glutenins. Glutenins mainly confer dough elasticity and gliadins dough extensibility essential for bread-making [3,4]. Glutenins are classed as high molecular weight-glutenin subunits (HMW-GS) encoded at Glu-1 loci and low molecular weight-glutenin subunits (LMW-GS) encoded at Glu-3 loci. HMW-GS are further subdivided into high $M_{\mathrm{r}}$ x-type and low $M_{\mathrm{r}} \mathrm{y}$-type subunits. Tightly linked pairs of genes encoding one x-type and one y-type subunit are found on the 1A, 1B and 1D chromosomes of hexaploid bread wheat [5].

Both $\mathrm{x}$ - and y-type HMW-GS have a large central elastomeric repetitive domain flanked by short non-repetitive $N$ - and $C$-terminal domains. According to results from circular dichroism (CD) spectroscopy and computer prediction from amino acid sequences, the $\mathrm{N}$ - and $\mathrm{C}$-terminal domains are probably both rich in $\alpha$-helices [6] and the dominant structural feature of the central repetitive domain is the presence of $\beta$-reverse turns [7]. The main difference between the two types of HMW-GS is that 18 residues of the $N$-terminal domain after residue 33 are deleted in x-type subunits, such that the domain is 81 to 89 residues long in x-type subunits but 104 residues long in y-type. In contrast, the $C$-terminal domain of both types is made up of 42 residues [8].

The distribution and number of cysteine residues in all three domains are of particular interest because disulfide bonds alter the polymeric structure and the conformation of the protein, which in turn is critical for the viscoelastic properties of the wheat dough $[9,10]$. All y-type subunits have five cysteines in their $\mathrm{N}$-terminal domain. Two of these residues are localized in the 18 -residue sequence that is deleted in x-type subunits, which therefore have no more than three cysteines in the $N$-terminal domain. HMW-GS $N$-terminal domains therefore have one (in $1 \mathrm{Bx} 14$ and $1 \mathrm{Bx} 20$ ), three (in $1 \mathrm{Bx} 7$, 1Bx17, all 1Dx and 1Ax subunits) or 5 (all y-type subunits) cysteine residues. All HMW-GS have only one cysteine residue in the $C$-terminal domain.

Cysteine residues are only present within the central repetitive domains in y-type subunits, but are not usually present in the equivalent domain in x-type subunits, although there are a few exceptions. Like y-type subunits, the central repetitive domain of the 1Dx5 subunit contains an additional cysteine residue. However, it is in a different position than cysteines in y-type subunits, being in the first repetitive block adjacent to the $N$-terminal domain [11]. Buonocore et al. [10] suggested that this "extra" cysteine residue might be the major factor explaining why this gluten subunit is associated with good quality. In the $1 \mathrm{Ax} 2{ }^{* \mathrm{~B}}$ subunit, an $\mathrm{x}$-type HMW-GS encoded at the Glu-A1 locus, there is a cysteine residue in the central domain in a different position compared to that in $1 \mathrm{Dx} 5$ [12].

As well as cysteine residues, the specific amino acid composition of HMW-GS is important in determining their elastic and polymeric behavior and thus the rheology and processing quality of wheat dough [13]. For example, the repetitive sequences rich in Gln residues form an extensive interchain array of hydrogen bonds, which makes wheat dough more elastic [14]. Tyrosine cross-links also influence the rheological properties of dough and gluten [15].

In general, the HMW-GS are the major genotypic determinants of dough strength and determine the suitability of the wheat for bread-making by conferring dough viscoelasticity [16]. The variability 
among the HMW-GS alleles has been repeatedly shown to influence bread-making quality [17]. The analysis of genetic variability of HMW-GS was initiated in the 1980s by Payne and Lawrence [18] when $20 \mathrm{HMW}-\mathrm{GS}$ alleles were identified by electrophoresis of glutenin proteins on acrylamide gels, namely 3 alleles at the Glu-A1 locus, 11 at the Glu-B1 locus and 6 at the Glu-D1 locus. Several other HMW glutenin alleles were later identified [16,19-23].

Analysis of the genetic diversity of 'Barbela', a bread wheat population cultivated in Portugal, revealed the existence of a new HMW-GS, named 1.1, encoded at the Glu-A1 locus. We previously found that this specific subunit is implicated in the high dough extensibility of flour from some 'Barbela' wheat lines [21], with alveograph L values up to $224 \mathrm{~mm}$ (G. Igrejas, unpublished data). This contrasts with other data showing that the $1 \mathrm{Ax} 1.1$ allelic effect on dough extensibility is similar to the effect of the 1Ax1 subunit, and suggests that the rheological data may be influenced by the environment in which experiments are carried out. However, there are also data from a series of crosses between 'Barbela' wheat lines encoding the subunit 1.1 and other bread wheat varieties showing that different HMW-GS combinations influence wheat quality differently [24].

Here we report the sequence of a new x-type HMW-GS encoded at the Glu-A1 locus and the impact of specific differences in the central repetitive domain on the technological value of the resulting wheat flour.

\section{Results and Discussion}

Glutenin subunits of the 'Barbela 28' wheat line were compared to those from other wheat lines and varieties with identical alleles encoded at Glu-Bl and Glu-D1 loci by sodium dodecyl sulfate-polyacrylamide gel electrophoresis (SDS-PAGE), shown in Figure 1.

The wheat line 'PI 355' was kindly provided by Q. Y. Li who reported the presence of subunit 1.1 in Triticum turgidum ssp. Dicoccum [25]. There is no band in the profile of the 'PI 355' sample with the same electrophoretic mobility as the 1.1 subunit found in 'Barbela 28'. The band with the least mobility in the 'PI 355' line has an apparent molecular weight very similar to subunit 1 found in 'Carala' and 'Konini'. To avoid confusion, it should be noted that the HMW-GS named 1.1 encoded at the Glu-A1 locus was described first in an analysis of genetic diversity of wheat grown in Portugal [21]. As expected the '211 12 04' wheat line shows an unusual x-type allele encoded at the Glu-A1 locus, named Ax2*[26]. The SDS-PAGE shows that HMW-GS 1.1 is less mobile than HMW-GS 1, with an apparent molecular weight between those of subunits 1Dx2.2 and 1Ax1.

The HMW-GS detected by SDS-PAGE were further identified by two-dimensional electrophoresis (2-DE), i.e., isoelectric focusing (IEF) followed by SDS-PAGE. The 2-DE profile of the 'Barbela 28' wheat line is showed in Figure 2.

All subunits analyzed were distinctly separated and most formed a single spot on the 2-DE gel. Comparison with other 2-DE patterns, and in particular with the 'Carala' pattern, revealed that subunit 1.1 in 'Barbela 28' was clearly distinguishable from subunit 1. 
Figure 1. Reduced and alkylated glutenin subunit patterns of selected bread wheat accessions analyzed by SDS-PAGE using a $12 \%$ gel for both high molecular weight-glutenin subunits (HMW-GS) and low molecular weight-glutenin subunits (LMW-GS). The HMW-GS are labeled according to the nomenclature of Payne and Lawrence [18]. Lane 1, 'Fukihokomugi'; lane 2, 'Barbela 28'; lane 3, 'PI 355'; lane 4, 'Carala'; lane 5, '211 12 04'; lane 6, '13-21'; lane 7, 'Konini'. The arrowhead points to the Glu-A1 allele 1.1. Sizes (in daltons) of protein molecular weight markers are shown on the left.

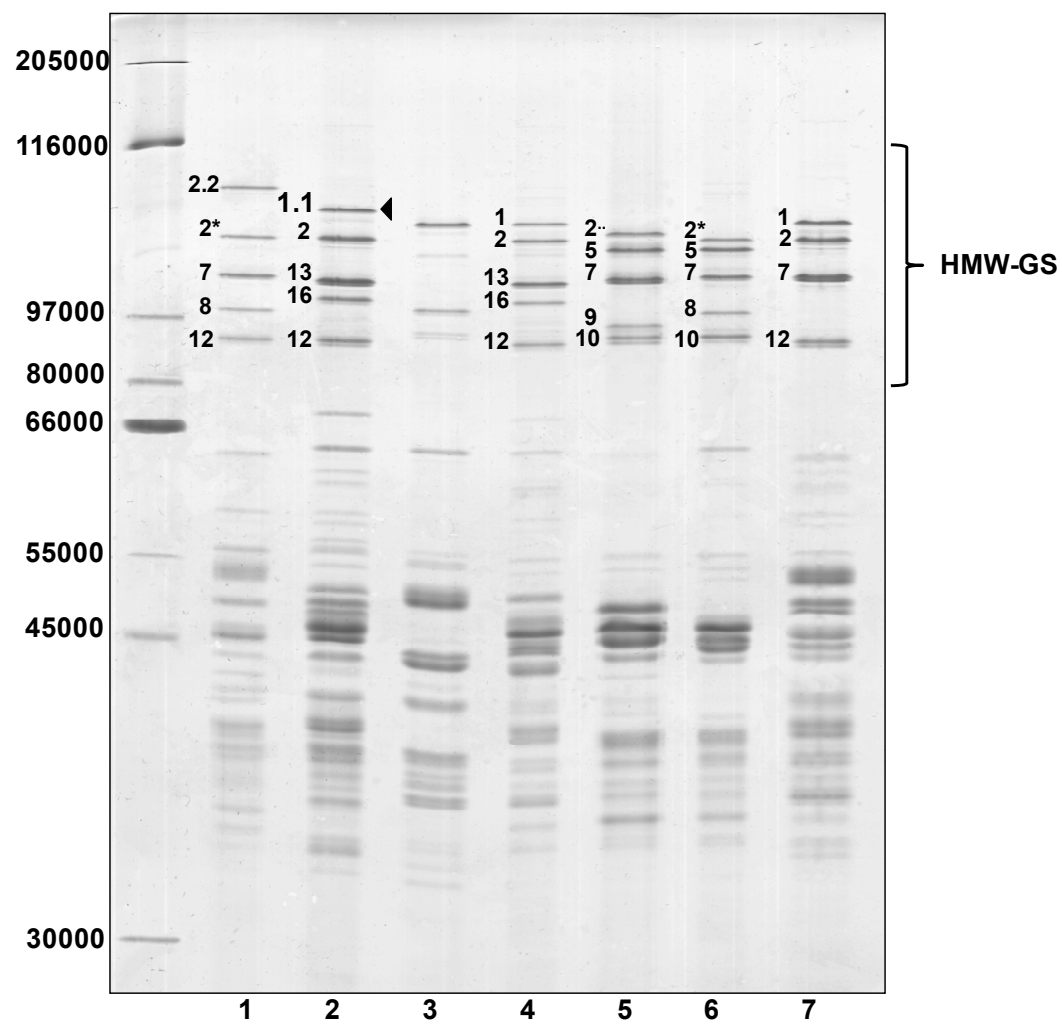

Figure 2. Two-dimensional electrophoresis pattern (IEF $\times$ SDS-PAGE) of the HMW-GS of wheat line 'Barbela 28'. The arrowhead points to the Glu-A1 allele 1.1. One-dimensional SDS-PAGE separations are shown to the left of the two-dimensional separations.

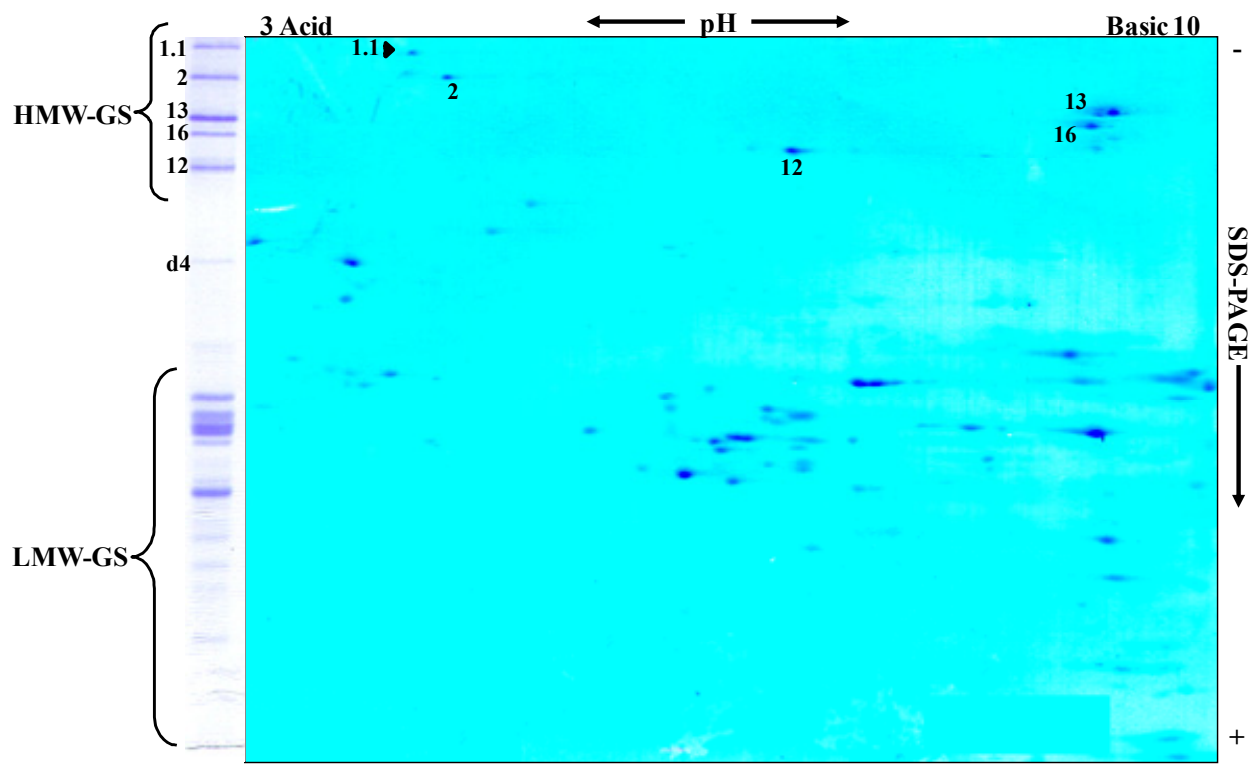


The following 2-DE patterns aid in distinguishing between different Glu-Al encoded alleles. First the line 'Barbela 28' is compared with the variety 'Carala' (Figure $3 \mathrm{~A}$ ) then with a 1:1 mixture of glutenin extracts from 'Carala' and 'Atlas 66' (Figure 3B).

Subunit 1.1 is clearly distinguished from subunit 1 , being slightly more acidic with a higher molecular weight. Subunit $2 *$ had a two-dimensional profile very different from subunits 1.1 and 1 , being more basic with a lower molecular weight. Subunit 2, present in each variety, overlapped precisely in all the 2-DE profiles.

Figure 3. Overlap of two-dimensional patterns of HMW-GS from 'Barbela 28' wheat line and other extracts (A) with the variety 'Carala' and (B) with an equal mixture from varieties 'Carala' and 'Atlas 66'. Subunits 1.1, 1,2 and $2^{*}$ are indicated.
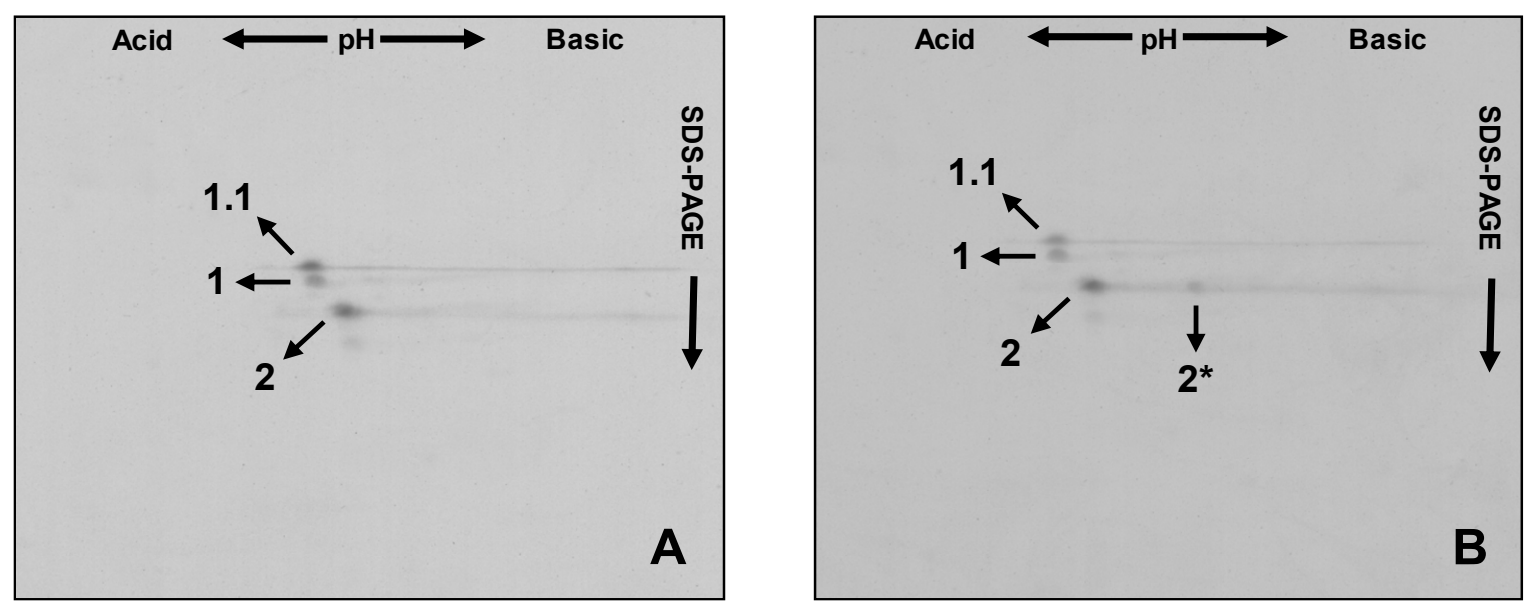

Mass spectrometry of trypchymo-digested peptides extracted from gel spots showed that subunit 1.1 (from the 'Barbela' wheat variety) and subunit 1 (from the 'Carala' wheat variety) are very similar (Figure 4). The latter subunit is strongly associated with good bread-making quality [27]. Subunit 1.1 peptide masses were used to search an NCBI non-redundant protein sequence database using the MASCOT tool and showed the greatest resemblance to subunit 1 (GenBank ${ }^{\circledR}$ accession number CAA43331.1). Of the 39 subunit 1.1 peptide masses, 20 values matched those of subunit 1 giving an identity score of 184 (with $27 \%$ of sequence coverage). In Figure 4B, a few $\mathrm{MH}+$ ion peaks, like one at $m / z$ 983.5233, are not shown because of the scale (34-41). Mass spectra were used at this stage to confirm that the isolated protein was indeed an x-type high molecular weight glutenin subunit encoded at the Glu-A1 locus. As $N$ - and $C$-terminal domains of the subunits $1 \mathrm{Ax} 1.1$ and $1 \mathrm{Ax} 1$ are very similar, data from mass spectra and from sequence databases were used together to design suitable primers.

Specific primers were designed to amplify the Glu-A1 locus from 'Barbela 28' genomic DNA, i.e., the gene encoding HMW-GS 1.1, and the amplified DNA was sequenced and analyzed. The 1Ax1.1 ORF is $2601 \mathrm{bp}$ long, which is slightly longer than the 2493-bp ORF reported for the gene that encodes subunit 1 [28] and is consistent with the higher molecular weight of subunit 1.1 found in gel electrophoresis (Figure 1). 
Figure 4. MALDI-TOF mass spectra of the two high molecular weight glutenin subunits 1Ax1 and 1Ax1.1. (A) Individual mass traces and (B) Overlaid mass traces.
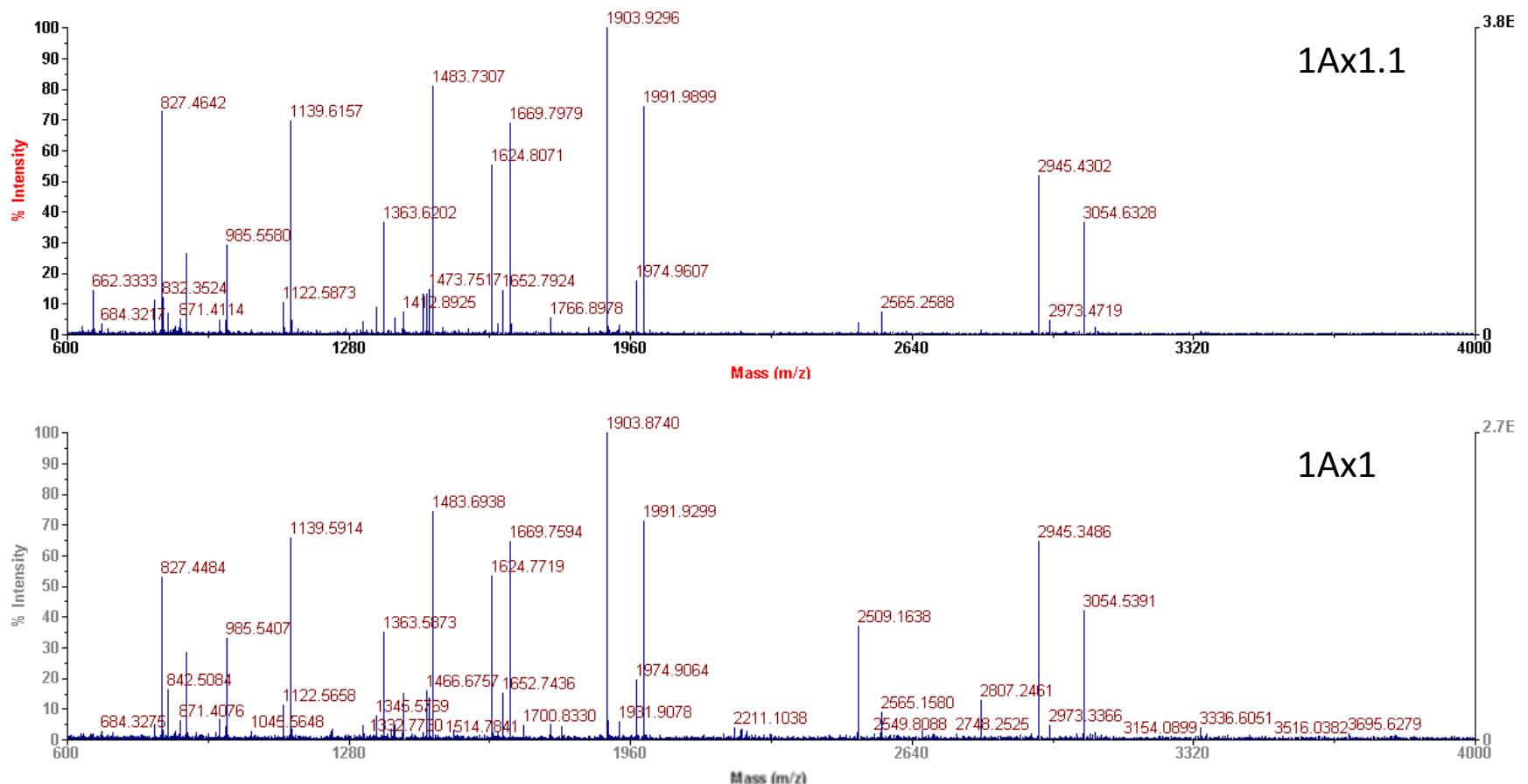

(A)

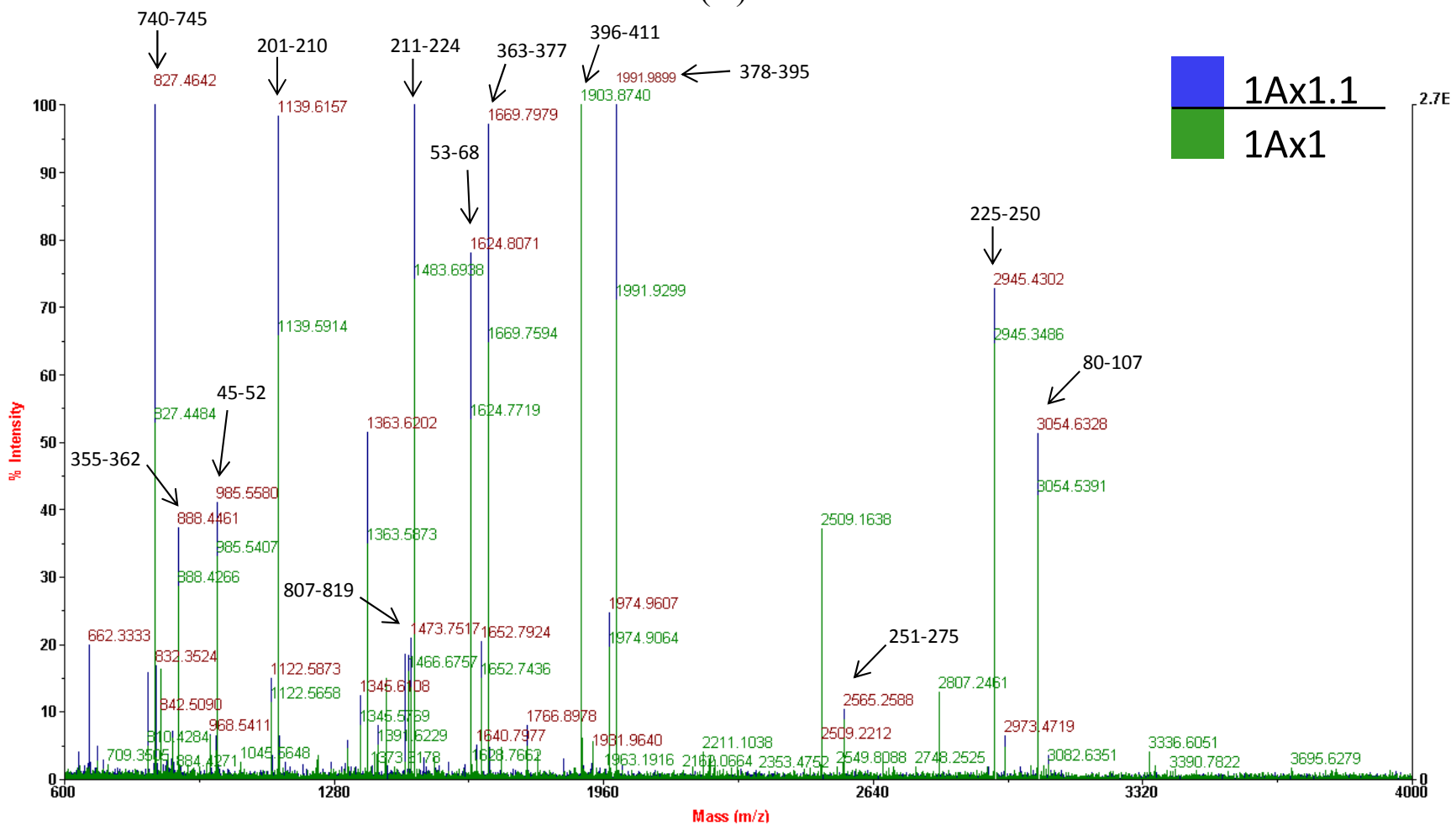

(B)

A high level of nucleotide identity (95\%) was found between coding sequences of $1 \mathrm{Ax} 1.1$ and 1Ax1 HMW-GS genes (GenBank ${ }^{\circledR}$ accession nos. JN172932.1 and X61009.1 respectively). The sequence alignment of the 1.1 and 1 subunit ORFs is shown in Figure 5. High levels of identity with other Glu-Al encoded alleles were also found, e.g., with the null allele and with $2 \cdot, 2 *$ and $2{ }^{* \mathrm{~B}}$ alleles

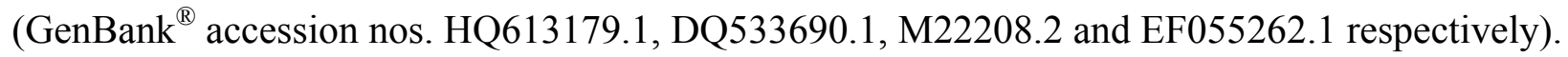


Figure 5. Alignment of $1 \mathrm{Ax} 1.1{\left(\mathrm{GenBank}^{\circledR}\right.}^{\circledR}$ accession no. JN172932.1) and $1 \mathrm{Ax} 1$ (GenBank ${ }^{\circledR}$ accession no. X61009.1) HMW-GS nucleotide coding sequences. The identity between the two sequences is $95 \%$.

\begin{tabular}{|c|c|c|}
\hline $\begin{array}{l}1 \mathrm{~A} \times 1.1 \\
1 \mathrm{Ax} 1\end{array}$ & AGACCGTCCAAAAATCTGTTTTACAAAGCTCCAATTGCTCCTTGCTTATCCAGCTTTTTT & 60 \\
\hline $\operatorname{Ax} 1.1$ & & \\
\hline $1 \mathrm{~A} \times 1$ & TGTGTTGGCAAACTACACTTTTTCAACCGATTTTGTTCTTCTCACACTTTCTTCTTAGGC & 120 \\
\hline$A \times 1.1$ & 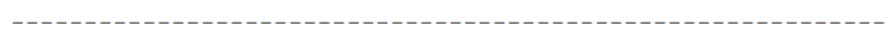 & \\
\hline $1 \mathrm{Ax} 1$ & TAAACAAACCTTACCGTGCACGCAGCCATGGTCCTGAATCTTCACCTCGTCCCTATAAAA & 180 \\
\hline $1 \mathrm{Ax} 1.1$ & 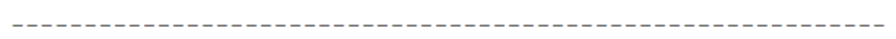 & \\
\hline $1 \mathrm{Ax} 1$ & GCCTAGCCAACCTTCACAATCTCTTCATCACCCACAACACCGAGCATCACAAACTAGAGA & 240 \\
\hline $1 \mathrm{Ax} 1.1$ & 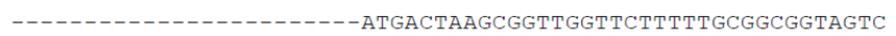 & 36 \\
\hline $1 \mathrm{Ax} 1$ & 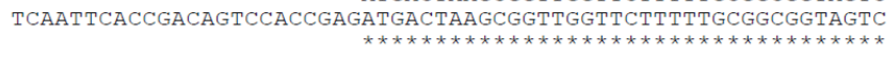 & 300 \\
\hline $1 \mathrm{Ax} 1.1$ & GTCGCCCTTGTGGCTCTCACCGCTGCTGAAGGTGAGGCCTCAGGGCAAGTACAGTGTGAG & 96 \\
\hline $1 \mathrm{Ax} 1$ & 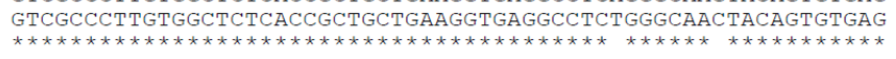 & 360 \\
\hline $1 \mathrm{Ax} 1.1$ & CGCGAGCTCCAAGAACACTCGCTTAAGGCATGCCGACAGGTCGTAGACCAGCAGCTCCGA & 156 \\
\hline $1 \mathrm{Ax} 1$ & 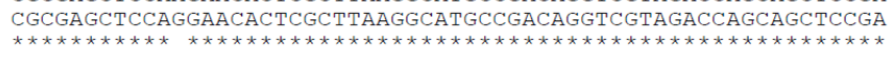 & 420 \\
\hline $1 \mathrm{~A} \times 1.1$ & GACGTTAGCCCCGAGTGCCAACCCGTCGGCGGCGGCCCGGTCGCGAGACAATATGAGCAG & 216 \\
\hline $1 \mathrm{~A} \times 1$ & 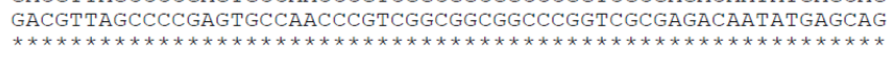 & 480 \\
\hline $1 \mathrm{~A} \times 1.1$ & CAAGTCGTGGTGCCGCCCAAGGGTGGATCTTTCTACCCCGGCGAGACCACGCCACCACAG & 276 \\
\hline $1 \mathrm{Ax} 1$ & 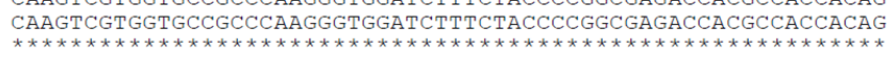 & 540 \\
\hline $1 \mathrm{Ax} 1.1$ & CAACTCCAACAAAGTATACTTTGGGGAATACCTGCACTACTAAGAAGGTATTACCTAAGT & 336 \\
\hline $1 \mathrm{Ax1}$ & 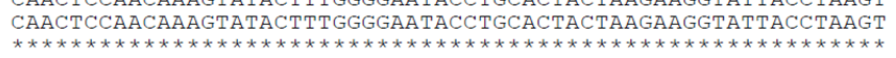 & 600 \\
\hline $1 \mathrm{Ax} 1.1$ & GTAACTTCTCCGCAACAGGTTTCATACTATCCAGGCCAAGCTTCTTCGCAACGGCCAGGA & 396 \\
\hline $1 \mathrm{Ax} 1$ & 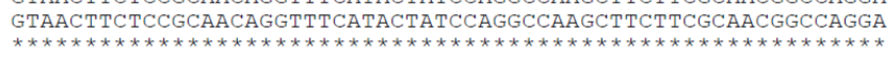 & 660 \\
\hline $1 \mathrm{Ax} 1.1$ & CAAGGTCAGCAGCCAGGACAAGGACAACAAGAATACTACCTAACTTCTCCGCAACAGTCA & 456 \\
\hline $1 \mathrm{Ax} 1$ & 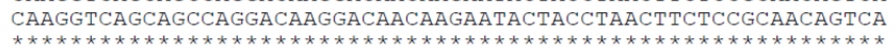 & 720 \\
\hline $1 \mathrm{Ax} 1.1$ & GGACAATGGCAACAACCGGGACAAGGGCAATCAGGGTACTACCCAACTTCTCCGCAGCAG & 516 \\
\hline $1 \mathrm{Ax} 1$ & 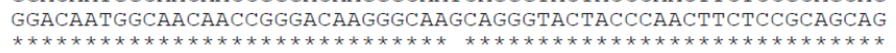 & 780 \\
\hline $1 \mathrm{Ax} 1.1$ & TCAGGACAAGAGCAACCAGGGTACTATCCAACTTCTCCATGGCAGCCAGAACAATTGCAA & 576 \\
\hline $1 \mathrm{Ax} 1$ & 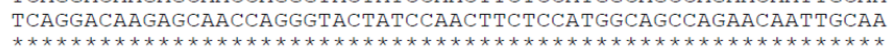 & 840 \\
\hline $1 \mathrm{Ax} 1.1$ & CAACCAACACAAGGGCAACAAAGACAGCAACCAGGACAAGGTCAGCAACTAAGACAAGGA & 636 \\
\hline $1 \mathrm{Ax} 1$ & 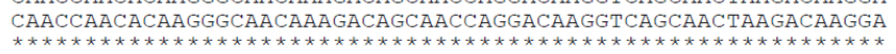 & 900 \\
\hline $\begin{array}{l}1 \mathrm{Ax} 1 \cdot 1 \\
1 \mathrm{Ax} 1\end{array}$ & 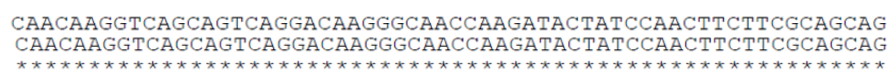 & $\begin{array}{l}696 \\
960\end{array}$ \\
\hline $1 \mathrm{Ax} 1.1$ & CCAGGACAATTGCAACAACTAGCACAAGGCCAACAAGGGCAGCAACCAGAACGAGGGCAA & 756 \\
\hline $1 \mathrm{Ax} 1$ & 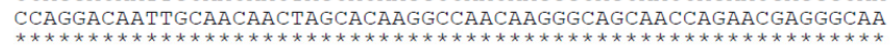 & 1020 \\
\hline $1 \mathrm{Ax} 1.1$ & CAAGGCCAACAGTCAGGACAAGGGCAACAACTAGGACAAGGGCAACAAGGTCAGCAGCCA & 816 \\
\hline $1 \mathrm{Ax} 1$ & $\begin{array}{l}\text { CAAGGCCAACAGTCAGGACAAGGGCAACAACTAGGACAAGGGCAACAAGGTCAGCAGCCA } \\
* * * * * * * * * * * * * * * * * * * * * * * * * * * * * * * * * * * * * * * * * * * * * * * * * * * * * * * * * * * * * *\end{array}$ & 1080 \\
\hline $1 \mathrm{Ax} 1.1$ & GGACAAAAGCAACAATCAGGACAAGGACAACAAGGGTACTACCCAATTTCTCCGCAACAG & 876 \\
\hline $1 \mathrm{Ax} 1$ & 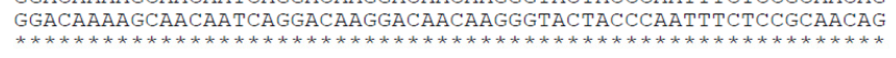 & 1140 \\
\hline $\begin{array}{l}1 \mathrm{Ax} 1.1 \\
1 \mathrm{Ax} 1\end{array}$ & 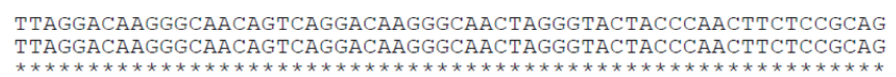 & $\begin{array}{l}936 \\
1200\end{array}$ \\
\hline $1 \mathrm{Ax} 1.1$ & CAGTCAGGACAAGGACAATCAGGATACTATCCAACTTCTGCGCAGCAGCCAGGACAATTG & 996 \\
\hline $10 \times 1$. & 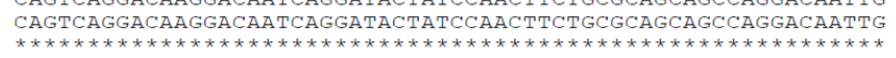 & 1260 \\
\hline $1 \mathrm{Ax} 1.1$ & CAACAATCAACACAAGAGCAGCAATTAGGACAAGAGCAACAAGATCAGCAATCAGGACAA & 1056 \\
\hline $1 \mathrm{Ax} 1$ & $\begin{array}{l}\text { CAACAATCAACACAAGAGCAGCAATTAGGACAAGAGCAACAAGATCAGCAATCAGGACAA } \\
* * * * * * * * * * * * * * * * * * * * * * * * * * * * * * * * * * * * * * * * * * * * * * * * * * * * * * * * * * * * * * * * * * * * *\end{array}$ & 1320 \\
\hline & GTCAACAGTCAGGACAAAGGCAACAAGATCAGCAGTCAGGACAAGGGCAG & 11. \\
\hline $1 \mathrm{Ax} 1$ & GGGCGACAAGGTCAACA GTCA GGACAAAGGCAACAAGATCAGCAGTCAGGACAAGGGCAG & 1380 \\
\hline
\end{tabular}


Figure 5. Cont.

\begin{tabular}{|c|c|c|}
\hline \multirow{2}{*}{$\begin{array}{l}1 \mathrm{Ax} 1.1 \\
1 \mathrm{Ax} 1\end{array}$} & CAACCGGGACAAAGGCAGCCAGGGTACTACTCAACTTCTCCGCAACAATTAGGACAAGGG & 1176 \\
\hline & 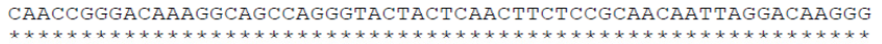 & 1440 \\
\hline $1 \mathrm{Ax} 1.1$ & СAACCAAGGTACTACCCAACTTCTCCGCAGCAGCCAGGACAAGAGCAGCAGCCAAGACAA & 1236 \\
\hline $1 \mathrm{Ax} 1$ & 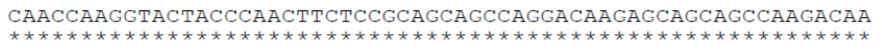 & 1500 \\
\hline $1 \pi \times 1.1$ & 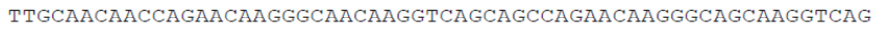 & 1296 \\
\hline $1 \mathrm{Ax} 1$ & 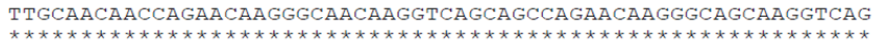 & 1560 \\
\hline $1 \mathrm{Ax} 1.1$ & 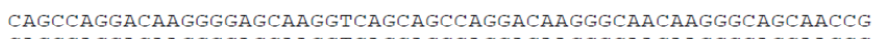 & 1356 \\
\hline $1 \mathrm{Ax} 1$ & 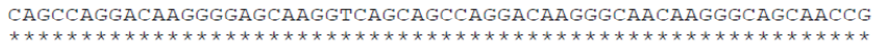 & 1620 \\
\hline $1 A \times 1.1$ & GGACAAGGGCAGCCAGGGTACTACCCA ACTTCTCCGCAGCAGTCGGGACAAGGGCAACCA & 1416 \\
\hline $1 \mathrm{~A} \times 1$ & $\begin{array}{l}\text { GGACAAGGGCAGCCAGGGTACTACCCAACTTCTCCGCAGCAGTCGGGACAAGGGCAACCA } \\
* * * * * * * * * * * * * * * * * * * * * * * * * * * * * * * * * * * * * * * * * * * * * * * * * * * * * * * * * * * * *)\end{array}$ & 1680 \\
\hline $1 \mathrm{Ax} 1.1$ & GGGTACTACCCAACTTCTCCACAGCAGTCAGGACAATTGCAACAACCAGCACAAGGGCAG & 1476 \\
\hline $1 \mathrm{Ax} 1$ & 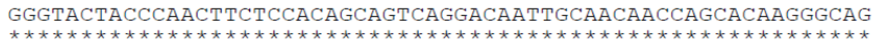 & 1740 \\
\hline $\begin{array}{l}1 \mathrm{Ax} 1 \cdot 1 \\
1 \mathrm{Ax} 1\end{array}$ & $\begin{array}{l}\text { CAACCAGGACAAGAGCAACAAGGTCAACAGCCAGGACAAGGGCAACAAGGTCAACAGCCA } \\
\text { CAACCAGGACAAGAGCAACAAGGTCAACAGCCAGGACAAGGGCAACAAGGTCAACAGCCA } \\
* * * * * * * * * * * * * * * * * * * * * * * * * * * * * * * * * * * * * * * * * * * * * * * * * * * * * * *\end{array}$ & $\begin{array}{l}1536 \\
1800\end{array}$ \\
\hline $1 \mathrm{Ax} 1.1$ & GGACAAGGGCAGCAACCGGGACAAGGGCAGCCAGGGTACTACCCAACTTCTCCGCAGCAG & 1596 \\
\hline $1 \mathrm{Ax} 1$ & $\begin{array}{l}\text { GGACAAGGGCAGCAACCGGGACAAGGGCAGCCAGGGTACTACCCAACTTCTCCGCAGCAG } \\
* * * * * * * * * * * * * * * * * * * * * * * * * * * * * * * * * * * * * * * * * * * * * * * * * * * * * * * * * * * * * * * *\end{array}$ & 1860 \\
\hline $1 \mathrm{Ax} 1.1$ & TCAGGACAAGAGCAACAGCTAGAACAATGGCAACAGTCAGGACAGGGGCAACCAGGGCAC & 1656 \\
\hline $1 \mathrm{Ax} 1$ & $\begin{array}{l}\text { TCAGGACAAGAGCAACAGCTAGAACAATGGCAACAGTCAGGACAGGGGCAACCAGGGCAC } \\
* * * * * * * * * * * * * * * * * * * * * * * * * * * * * * * * * * * * * * * * * * * * * * * * * * * * * * * * * * * *\end{array}$ & 1920 \\
\hline $1 \mathrm{Ax} 1.1$ & TACCCAACTTCTCCGTTGCAGCCAGGACAAGGGCAACCAGGGTACTACCCAACTTCTCCA & 1716 \\
\hline $1 \mathrm{Ax} 1$ & 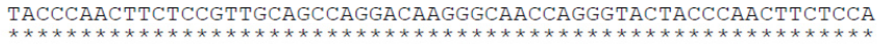 & 1980 \\
\hline $1 \mathrm{Ax} 1.1$ & CAACA GATAGGACAAGGGCAGCAGCCAGGACAATTGCAACAACCAACACAAGGGCAACAA & 1776 \\
\hline 1 Axl & 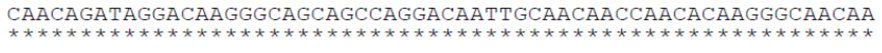 & 2040 \\
\hline $1 \mathrm{Ax} 1.1$ & GGGCAGCAACCAGGACAAGGGCAACAAGGTCAACAGCCAGGACAAGGGCAACAAGGTCAG & 1836 \\
\hline $1 \mathrm{Ax} 1$ & GGGCAGCAACCAGGACAAGGGCAACAAGGTCAACAGCCAGGACAAGGGCAACAAGGTCAG & 2100 \\
\hline $\begin{array}{l}1 \mathrm{~A} \times 1 \cdot 1 \\
1 \mathrm{Ax} 1\end{array}$ & $\begin{array}{l}\text { CAGCCAGGACAAGGGCAGCAACCAGGACAAGGGCAGCCAGGGTACTACCCAACTTCTTTG } \\
\text { CAGCCAGGACAAGGGCAGCAACCAGGACAAGGGCAGCCAGGGTACTACCCAACTTCTTTG } \\
* * * * * * * * * * * * * * * * * * * * * * * * * * * * * * * * * * * * * * * * * * * * * * * * * * * * * *\end{array}$ & $\begin{array}{l}1896 \\
2160\end{array}$ \\
\hline$\perp \mathrm{Ax} \perp . \perp$ & CAGCAGI'CAGGACAAGGGCAACAGCCAGGACAA'I'GGCAACAACCAGGACAAGGAC'I'ACCA & 1956 \\
\hline $1 \mathrm{AXI}$ & 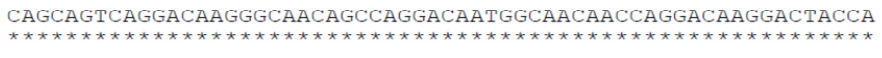 & 2220 \\
\hline $1 \mathrm{Ax} 1.1$ & GGGTACTACCCAACTTCTTCGTTGCAGCCAGAACAAGGGCAACAAGGGTACTACCCAACT & 2016 \\
\hline $1 \mathrm{~A} \times 1$ & 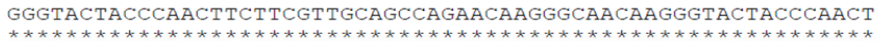 & 2280 \\
\hline $1 A \times 1.1$ & 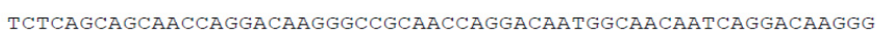 & 2076 \\
\hline $1 \mathrm{~A} \times 1$ & 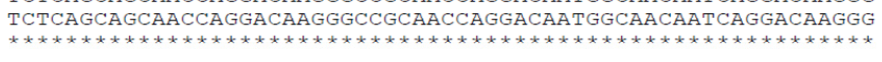 & 2340 \\
\hline $1 \mathrm{Ax} 1.1$ & САAСАAGGGTACTACССААСТTСТCСGCAGCAGTCAGGACAAGGGCAACAGCCAGGACAA & 2136 \\
\hline $1 \mathrm{Ar} 1$ & 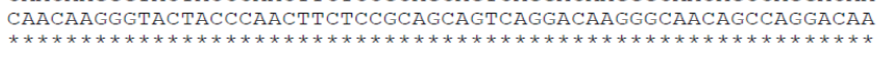 & 2400 \\
\hline $1 \mathrm{Ax} 1.1$ & TGGTTGCAACCAGGACAATGGCTGCAATCAGGGTACTACCTAACTTCTCCGCAGCAGTTA & 2196 \\
\hline $1 \mathrm{Ax} 1$ & 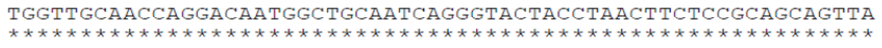 & 2460 \\
\hline $1 \mathrm{Ax} 1.1$ & 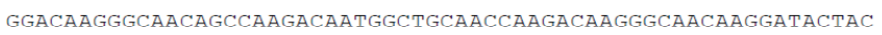 & 2256 \\
\hline $1 \mathrm{~A} \times 1$ & $\begin{array}{l}\text { GGACA AGGGCAACAGCCAAGACAATGGCTGCAACCAAGACAAGGGCAACAAGGATACTAC } \\
* * * * * * * * * * * * * * * * * * * * * * * * * * * * * * * * * * * * * * * * * * * * * * * * * * * * * * * *\end{array}$ & 2520 \\
\hline $1 \mathrm{Ax} 1.1$ & CCAACTTCTCCGCAGCAGTCAGGACAAGGGCAACAATTAGGACAAGGGCAACAAGGATAC & 2316 \\
\hline $1 \mathrm{Ax} 1$ & 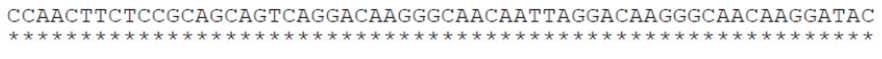 & 2580 \\
\hline $1 \mathrm{Ax} 1.1$ & TACCCAACTTCTCCGCAGCAGTCAGGACAAGGGCAACAAGGATACTACCCAACTTCTCCG & 2376 \\
\hline $1 \mathrm{Ax} 1$ & $\begin{array}{l}\text { TACCCAACTTCTCCGCAGCAGTCAGGACAAGGGCAACAAGGCTACGAC--AGC---CCA } \\
* * * * * * * * * * * * * * * * * * * * * * * * * * * * * * * * * * * * * * * * * * * * * * * * * * \\
* * *\end{array}$ & 2634 \\
\hline $1 A \times 1.1$ & CAGCA-GTCAGGACAAGGGCAACAA----TTAGGACAAGGGCAACAAGGATACTACCCAA & 2431 \\
\hline $1 \mathrm{Ax} 1$ & 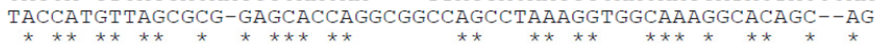 & 2691 \\
\hline $1 \mathrm{Ax} 1.1$ & CTT-CTCCGCAGCAGTCAG-GACAAG--GGCAACAAGGCTACGACAGCCCAT--ACCATG & 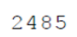 \\
\hline $1 \mathrm{Ax} 1$ & CTCGCGGCACAGCTGCCGGCAATGTGCCGGCTAGAGGGCGGCGACGCATTGTTGGCCA-G & 275 \\
\hline & 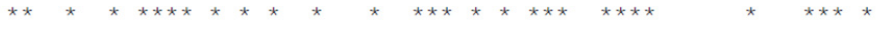 & \\
\hline $1 \mathrm{Ax} 1.1$ & $\mathrm{CAA}--\mathrm{AGGCACAGCAGCT- \textrm {CG }}$ & 25 \\
\hline $1 \mathrm{AxI}$ & 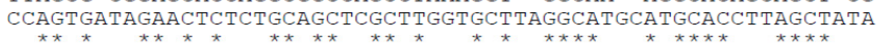 & 2810 \\
\hline $1 \mathrm{~A} \times 1.1$ & 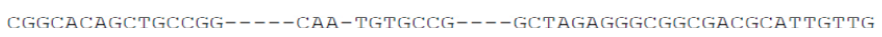 & 2.589 \\
\hline $1 \mathrm{Ax} 1$ & CAATAAATGTGGCGTGTGTTCAAGTTTTTCATGTAACTAATGTAAAGCCCAGTAATGATG & 2870 \\
\hline & $* \quad * * * * * *$ & \\
\hline & GCCAGCCAGTGA------- 2601 & \\
\hline $1 \mathrm{AXN}$ & - CAA--AATGAAAAGCTT $_{\star \star \star} 2885$ & \\
\hline
\end{tabular}


Several differences were found between the two sequences. As well as the 1.1 ORF having 108 more base pairs than the ORF of subunit 1, there are 46 single-base, 12 two-base, 9 three-base, 1 four-base and 1 six-base substitutions. There are also 1 single-base, 4 two-base and 1 four-base insertions and 6 single-base, 4 two-base, 2 four-base and 1 five-base deletions.

The HMW-GS 1.1 peptide sequence is 866 amino acids long, and HMW-GS 1 is 830 amino acids long. The predicted amino acid composition of $N$ - and $C$-terminal domains of subunit 1.1 is identical to subunit 1 , as expected from mass spectrometry data. All the subunit 1 cysteines were conserved in subunit 1.1 and no extra cysteine residue was found in the central repetitive domain of 1.1. This indicates that the patterns of intra- and inter-chain disulfide bonds are identical for both proteins.

In addition, two substitutions in highly conserved residues were found at positions +29 and +163 in the $N$-terminal and central repetitive domains, respectively (Figure 6).

Figure 6. Alignment of the predicted primary structure of 1Ax1.1 and 1Ax1 HMW-GS. The dashes indicate the deletion of 36 amino acids at the end of the central repetitive domain at position +771 relative to the $1 \mathrm{Ax} 1.1$ sequence. Substitutions are indicated by colons.

\begin{tabular}{|c|c|c|}
\hline $1 \mathrm{Ax} 1.1$ & MTKRLVLFAAVVVALVALTAAEGEASGQVQCERELQEHSLKACRQVVDQQLRDVSPECQP & 60 \\
\hline $1 \mathrm{Ax} 1$ & 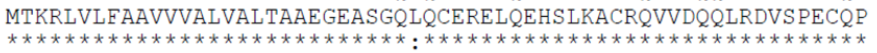 & 60 \\
\hline $1 \mathrm{Ax} 1.1$ & VGGGPVARQYEQQVVVPPKGGSFYPGETTPPQQLQQSILWGI PALLRRYYLSVTS PQQVS & 120 \\
\hline $1 \mathrm{Ax} 1$ & 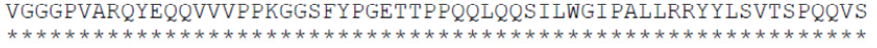 & 120 \\
\hline $1 \mathrm{~A} \times 1.1$ & YYPGQASSQRPGQGQQPGQGQQEYYLTSPQQSGQWQQPGQGQSGYYPTSPQQSGQEQPGY & 180 \\
\hline $1 \mathrm{Ax} 1$ & 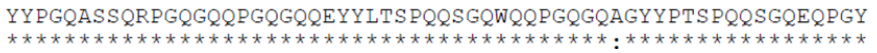 & 180 \\
\hline $\begin{array}{l}1 \mathrm{Ax} 1.1 \\
1 \mathrm{Ax} 1\end{array}$ & 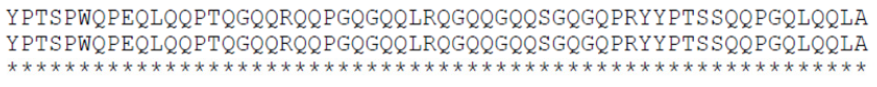 & $\begin{array}{l}240 \\
240\end{array}$ \\
\hline $\begin{array}{l}1 \mathrm{Ax} 1.1 \\
1 \mathrm{Ax} 1\end{array}$ & 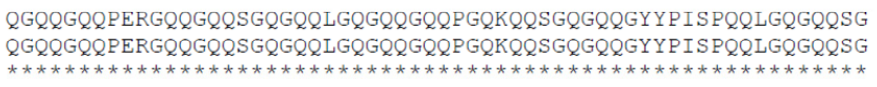 & $\begin{array}{l}300 \\
300\end{array}$ \\
\hline $\begin{array}{l}1 \mathrm{Ax} 1.1 \\
1 \mathrm{Ax} 1\end{array}$ & 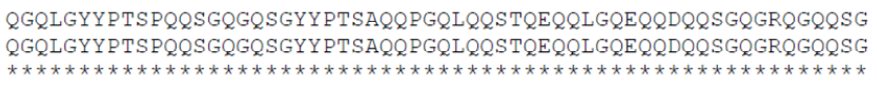 & $\begin{array}{l}360 \\
360\end{array}$ \\
\hline $\begin{array}{l}1 \mathrm{Ax} 1.1 \\
1 \mathrm{Ax} 1\end{array}$ & 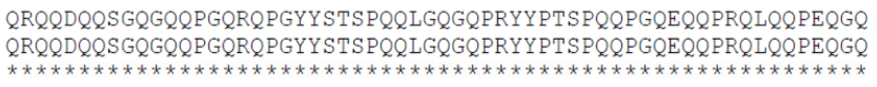 & $\begin{array}{l}420 \\
420\end{array}$ \\
\hline $\begin{array}{l}1 \mathrm{Ax} 1.1 \\
1 \mathrm{Ax} 1\end{array}$ & 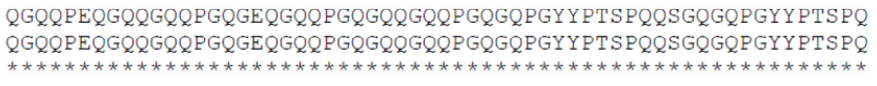 & $\begin{array}{l}480 \\
480\end{array}$ \\
\hline $\begin{array}{l}1 \mathrm{Ax} 1.1 \\
1 \mathrm{Ax} 1\end{array}$ & 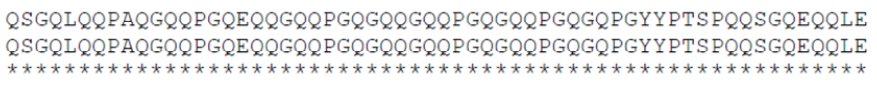 & $\begin{array}{l}540 \\
540\end{array}$ \\
\hline $\begin{array}{l}1 \mathrm{Ax} 1.1 \\
1 \mathrm{Ax} 1\end{array}$ & 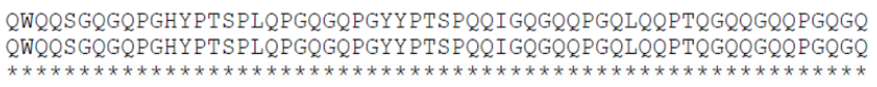 & $\begin{array}{l}600 \\
600\end{array}$ \\
\hline $\begin{array}{l}1 \mathrm{Ax} 1.1 \\
1 \mathrm{Ax} 1\end{array}$ & 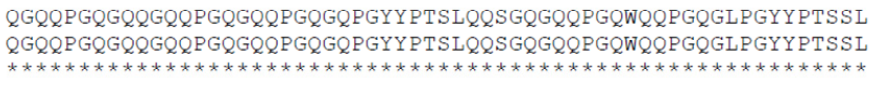 & $\begin{array}{l}660 \\
660\end{array}$ \\
\hline $\begin{array}{l}1 \mathrm{Ax} 1.1 \\
1 \mathrm{Ax} 1\end{array}$ & 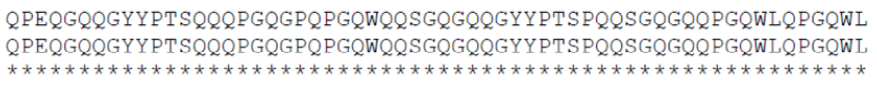 & $\begin{array}{l}720 \\
720\end{array}$ \\
\hline $\begin{array}{l}1 \mathrm{Ax} 1.1 \\
1 \mathrm{Ax} 1\end{array}$ & 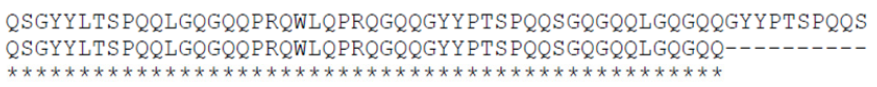 & $\begin{array}{l}780 \\
770\end{array}$ \\
\hline $\begin{array}{l}1 \mathrm{Ax} 1.1 \\
1 \mathrm{Ax} 1\end{array}$ & 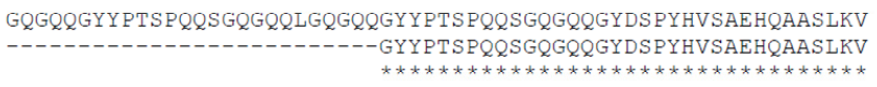 & $\begin{array}{l}840 \\
804\end{array}$ \\
\hline $\begin{array}{l}1 \mathrm{~A} \times 1.1 \\
1 \mathrm{Ax} 1\end{array}$ & $\begin{array}{l}\text { AKAQQLAAQLPAMCRLEGGDALLASQ } 866 \\
\text { AKAQQLAAQLPAMCRLEGGDALLASQ } 830 \\
\star * * * * * * * * * * * * * * * * * * * * * * *\end{array}$ & \\
\hline
\end{tabular}


In the central domain, there are some repeats based on combinations of motifs of tripeptides, like GQQ, hexapeptides, like PGQGQQ and PGQLQQ, and nonapeptides, like LRQGQQGQQ. The tripeptides also appear in tandem with hexapeptides, forming nine-residue motifs, like PGQGQQGQQ. Some nonapeptides are interspersed with hexapeptides, forming 15-residue motifs, like PGQGQQLRQGQQGQQ, while the hexapeptides occur in tandem arrays. The presence of numerous glutamine $(\mathrm{Q})$ residues should be noted, as these amino acids are involved in determining the elastic properties of wheat dough.

The insertion of 36 amino acids at the end of the central repetitive domain at position +771 in 1Ax1.1 (Figure 6) leads to some changes in the repetitive motifs compared with HMW-GS 1, namely the tripeptide GQQ, hexapeptide LGQGQQ and nonapeptide GYYPTSPQQ.

Comparing the MS data to the peptide sequence, we conclude that it was not possible to distinguish the two sequences by MALDI PMF either for the matched sequences or the unmatched masses. This is because almost all the peptides likely originate from tryptic cleavages. Peptide masses corresponding to fragments with any of the three differences found in the subunit 1Ax1.1 peptide (the two substitutions and the 36-aa insertion; Figure 6) were not detected.

Similar results regarding sequence coverage were reported by Qian et al. [29], who also reported difficulties in discriminating between two HMW-GS encoded at the Glu-A1 locus (1Ax1 and 1Ax2*). Previously, Hickman et al. [30] indicated that HMW subunits were not suitable for MS measurement because of poor resolution and even with individual proteins, the spectral peaks were broad. The relatively low frequencies of arginine and lysine residues in these proteins, which gives rise to mainly large tryptic peptides, and the close sequence similarity within this group of subunits, are consistently referred to as the main problems in characterizing HMW-GS by a MALDI PMF approach [29,31].

As reported elsewhere [29,31], we also attempted to measure the $M_{\mathrm{r}}$ of intact HMW-GS 1 and 1.1. The predicted masses of the intact subunits identified above, based on published DNA sequences, are in good agreement with the observed $\mathrm{m} / \mathrm{z}$ values obtained by linear MALDI-TOF (Table 1). The difference was less than $0.5 \%$ for both subunits, which is not significant if we consider the mass of the HMW-GS under analysis. Moreover, if we compare our results with previous data estimating the $M_{\mathrm{r}}$ of HMW-GS, this value is sufficient to be able to discriminate between the two subunits.

Table 1. Comparison of HMW-GS molecular masses deduced from coding gene sequences with those determined by MALDI-TOF-MS.

\begin{tabular}{|c|c|c|c|c|c|c|}
\hline \multirow{2}{*}{ HMW-GS } & \multicolumn{2}{|c|}{ Deduced $M_{\mathrm{r}}$ from coding gene } & \multicolumn{2}{|c|}{$M_{\mathrm{r}}$ by MALDI-TOF-MS } & \multicolumn{2}{|c|}{ Variation } \\
\hline & Origin & Mass (Da) & Origin & Mass (Da) & Difference (Da) & Error $(\%)$ \\
\hline $1 \mathrm{Ax} 1$ & 'Hope' & 87,678 & 'Carala' & 87,859 & -181 & 0.21 \\
\hline $1 \mathrm{Ax} 1.1$ & 'Barbela 28' & 91,508 & 'Barbela 28' & 91,858 & -350 & 0.38 \\
\hline
\end{tabular}

Analyzing old varieties or landraces has led to the identification of unusual allelic variants, some of which are already being incorporated into the genomes of current commercial wheat. Here we aimed to identify and characterize this unusual subunit. Proteomics was used to clearly distinguish subunit 1.1 from other subunits encoded at the Glu-Al locus like subunits 1, 2, 2*. In 2-DE, HMW-GS 1.1 migrates as a slightly more acidic protein than HMW-GS 1, although based on predictions from the 
primary sequence, the $\mathrm{pI}$ of both subunits should theoretically be 5.7. This makes subunit 1.1 the most acidic HMW-GS encoded at Glu-A1 locus reported so far (Figure 3).

The 1.1 ORF is predicted to encode a protein sequence with a $M_{\mathrm{r}}$ of 93,649 Da (or 91,508 Da for the mature protein), the highest $M_{\mathrm{r}}$ reported for an HMW-GS encoded by the Glu-Al locus. According to Don et al. [32], the very high molecular weight fraction of glutenins is considered important in determining wheat flour quality. HMW-GS are essential for the formation of glutenin macropolymer (GMP). Thus, the very high molecular weight of subunit 1.1 may have positive effects on the formation and size of the GMP. However, the presence or absence of the 1Ax1 subunit hardly affects the quantity of GMP present [32]. It is possible that subunit 1.1 has a similar or stronger positive effect on the formation of GMP. GMP and its rheological properties are widely reported to be good quality predictors [33-35]. Moreover, Weegels et al. [34] reported that GMP content is strongly related to the maximum resistance $\left(\mathrm{R}_{\max }\right)$ and extensibility of dough.

The insertion of 36 amino acids at the end of the central repetitive domain leads to some differences in the HMW-GS 1.1 repeat motifs compared with HMW-GS 1. The longer repetitive sequence may allow the formation of more hydrogen bonds and induce conformational differences. D'Ovidio et al. [36] suggested that the repetitive $\beta$-turn motifs in the central domain of HMW-GS explain their anomalously slow migration in SDS-PAGE. This might also explain the distinct difference in mobility of subunits $1 \mathrm{Ax} 1.1$ and $1 \mathrm{Ax} 1$, not only by the difference in mass $\left(3.8 \mathrm{kDa}\right.$ difference in theoretical $\left.M_{\mathrm{r}}\right)$ but also by differences in the number of repetitive domains in the protein secondary structure.

Preliminary technological tests were conducted to identify potential differences in flour quality conferred by 1Ax1.1 and 1Ax1 HMW-GS. Common tests used to evaluate wheat technological quality showed no significant differences between 1Ax1.1 and 1Ax1 (Table 2). This result is consistent with the identical patterns of intra- and inter-chain disulfide bonds that both proteins presumably form.

Table 2. Analysis of the impact of the allelic variation on quality parameters in wheat lines containing either $1 \mathrm{Ax} 1.1$ or $1 \mathrm{Ax} 1 \mathrm{HMW}-\mathrm{GS}$. Quality parameters are kernel protein content, grain hardness, tenacity (P), extensibility (L), deformation energy (W), tenacity/extensibility ratio $(\mathrm{P} / \mathrm{L})$ and elasticity index $(\mathrm{Ie})$.

\begin{tabular}{|c|c|c|c|c|c|c|c|c|}
\hline \multicolumn{2}{|c|}{ Comparison of Means } & \multicolumn{5}{|c|}{ Alveograph Parameters } & \multirow[b]{2}{*}{ Protein Content \% dm } & \multirow[b]{2}{*}{ GrainHardness } \\
\hline Location & $\begin{array}{c}\text { Genotype } \\
(G l u-A 1 ; G l u-B 1)\end{array}$ & $\mathbf{P} \mathbf{m m H}_{2} \mathbf{O}$ & $\mathbf{L} \mathbf{~ m m}$ & $\mathbf{W} 10^{-4} \mathrm{~J}$ & 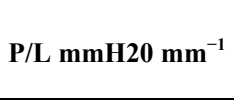 & Ie & & \\
\hline \multirow{4}{*}{ Portugal } & $1 ; 7+8$ & 44.8 & 101.3 & 80.3 & 0.5 & 25.1 & 15.0 & 53.7 \\
\hline & $1.1 ; 7+8$ & 51.7 & 110.7 & 114.4 & 0.5 & 27.6 & 14.2 & 35.6 \\
\hline & $1 ; 13+16$ & 63.8 & 143.4 & 184.0 & 0.4 & 35.2 & 14.3 & 23.0 \\
\hline & $1.1 ; 13+16$ & 57.8 & 119.6 & 141.1 & 0.5 & 30.9 & 15.3 & 41.9 \\
\hline \multirow{4}{*}{ France } & $1 ; 7+8$ & 70.4 & 208.6 & 295.1 & 0.33 & 44.7 & 17.5 & 77.0 \\
\hline & $1.1 ; 7+8$ & 72.6 & 181.8 & 243.1 & 0.40 & 37.0 & 14.8 & 49.7 \\
\hline & $1 ; 13+16$ & 93.5 & 148.8 & 370.5 & 0.63 & 50.2 & 14.8 & 49.1 \\
\hline & $1.1 ; 13+16$ & 70.4 & 131.8 & 224.9 & 0.53 & 42.7 & 15.1 & 60.7 \\
\hline
\end{tabular}

The Glu-Al allelic comparisons were performed using different sister lines that had different Glu-B1 alleles (either 7-8 or 13-16) yet were homogenous for Glu-D1, Glu-A3, Glu-B3 and Glu-D3 loci. No consistent differences were associated with subunit 1.1 or 1 . However, significant 
environmental effects were associated to the location where wheat was grown, either France or Portugal. The highest values were found in France for the alveograph parameters P, L W, Ie, and for protein content and grain hardness.

D'Ovidio et al. [37] reported an unusually large insertion of $561 \mathrm{bp}$ within the repetitive domain of a functional HMW glutenin gene encoded at Glu-Dl locus. Later, He et al. [38] studied the relationship between the subunit size and the effect on dough mixing properties of flour made from wheat lines transformed with a gene encoding an extended form of subunit 1Dx5. They found that none of the transgenic lines expressing the extended form of the 1Dx5 subunit showed the "overstrong" mixing properties exhibited by transgenic lines expressing the wild type 1Dx5 transgene. Similarly, in this work we demonstrated that the larger size of the subunit 1Ax1.1 and modification of the repetitive motifs did not produce a significant alteration in the rheology of dough. However, additional tests, including bread-making tests, are needed with subunit $1 \mathrm{Ax} 1.1$ expressed in different genetic backgrounds to ascertain whether this new allele has a positive impact on the size of polymers formed in wheat flour.

\section{Experimental Section}

\subsection{Plant Materials}

The 'Barbela' wheat population used was from the germplasm bank of the University of Trás-os-Montes and Alto Douro (UTAD), Vila Real, Portugal. Line 28 of 'Barbela' wheat was used throughout the study. The other germplasm used came from the germplasm bank of Institut National de la Recherche Agronomique (INRA), Clermont-Ferrand, France. The varieties used and their respective Glu-1 alleles are listed in Table 3.

Table 3. List of wheat varieties used in this study with their respective Glu-1 alleles encoding the HMW-GS.

\begin{tabular}{|c|c|c|c|c|c|c|}
\hline \multirow{2}{*}{ Variety } & \multicolumn{2}{|c|}{ Glu-A1 } & \multicolumn{2}{|c|}{ Glu-B1 } & \multicolumn{2}{|c|}{ Glu-D1 } \\
\hline & Allele & HMW-GS & Allele & HMW-GS & Allele & HMW-GS \\
\hline 'Fukihokomugi' & $\mathrm{b}$ & $2 *$ & $\mathrm{~b}$ & $7+8$ & $\mathrm{f}$ & $2.2+12$ \\
\hline 'Barbela 28' & $*$ & 1.1 & $\mathrm{f}$ & $13+16$ & $\mathrm{a}$ & $2+12$ \\
\hline 'PI 355' & $*$ & $*$ & $*$ & $*$ & $*$ & $*$ \\
\hline 'Carala' & a & 1 & $\mathrm{f}$ & $13+16$ & $\mathrm{a}$ & $2+12$ \\
\hline '211.12014' & $\mathrm{f}$ & $2 \cdot \cdot$ & $\mathrm{c}$ & $7+9$ & $\mathrm{~d}$ & $5+10$ \\
\hline ' $13-21$ ' & $\mathrm{b}$ & $2^{*}$ & $\mathrm{~b}$ & $7+8$ & $\mathrm{~d}$ & $5+10$ \\
\hline 'Konini' & $\mathrm{a}$ & 1 & $\mathrm{a}$ & 7 & $\mathrm{a}$ & $2+12$ \\
\hline 'Atlas 66' & $\mathrm{b}$ & $2 *$ & $\mathrm{f}$ & $13+16$ & $\mathrm{a}$ & $2+12$ \\
\hline
\end{tabular}

Other wheat lines and varieties with the same alleles encoded at Glu-B1 and Glu-D1 loci as in 'Barbela' were used as controls. For the two-dimensional analysis, the varieties 'Carala' and 'Atlas 66' were selected as controls as they have the same composition as 'Barbela' at Glu-B1 and Glu-D1 loci. 
For mass spectrometry analysis, the protein spots correspond to HMW-GS 1Ax1.1 from 'Barbela 28' and 1Ax1 from 'Carala'.

For analysis of the Glu-Al gene, in addition to 'Barbela 28' genomic DNA, genomic DNA from Triticum urartu (A genome), Triticum speltoides (B genome) and Triticum tauchii (D genome), and of the Triticum aestivum L. 'Chinese Spring' variety was used to verify the specificity of amplification of the Glu-Al locus in the 'Barbela 28' wheat line.

The different 'Barbela 28' sister lines carrying either subunit 1.1 or subunit 1 with either Glu-B1-encoded subunit 13-16 or subunit 7-8 were grown in two locations (in Vila Real, Portugal and in Clermont-Ferrand, France) in the year 2010-2011. At least two sister lines each having identical HMW-GS and LMW-GS composition were grown in the two locations with conventional fertilization and full fungicide protection.

\subsection{Technological Tests}

Each sample of flour from a specific wheat genotype was analyzed in duplicate and in two different locations: Vila Real, Portugal and Clermont-Ferrand, France.

Grain was milled using a Cyclotec lab mill (Tecator) to produce wholemeal. Flour protein content and kernel hardness were estimated on a wholemeal sample by near infrared reflectance (NIR-Percon Inframatic 8620) according to AACC (1995) Approved Methods 39-11 and 39-70A, respectively.

The Alveograph parameters such as tenacity $(\mathrm{P})$, extensibility $(\mathrm{L})$, the deformation energy or dough strength $(\mathrm{W})$, tenacity/extensibility ratio $(\mathrm{P} / \mathrm{L})$ and elasticity index (Ie) were determined according to the ICC standard $\mathrm{N}^{\circ} 121$.

\subsection{Electrophoresis}

HMW-GS were extracted according to the sequential method of Singh et al. [39] with some modifications. The HMW-GS present in the pellet were reduced and alkylated in a 50\% propanol solution with $1 \%$ dithiothreitol and $2.5 \%$ iodoacetamide, respectively. Glutenin subunits were separated in a resolving gel $(12.52 \% \mathrm{~T}$ and $0.97 \% \mathrm{C})$. The gels were stained with Coomassie blue and alleles were identified by referring to known alleles in control genotypes. The nomenclature of the HMW-GS corresponds to the terminology used by Payne and Lawrence [18].

Two-dimensional electrophoresis (2-DE) of HMW-GS was carried out as previously described [40]. Briefly the HMW-GS fraction obtained was added to extraction solution containing 4\% CHAPS, $7 \mathrm{M}$ urea, $2 \mathrm{M}$ thiourea, 1\% IPG buffer, $20 \mathrm{mM}$ DTT and milliQ ultrapure water. The flour plus the extraction solution were vortexed, sonicated and centrifuged. The rehydration of strips for isoelectric focusing (IEF, pH 3-10) was carried out using the rehydration solution consisting of extraction solution with bromophenol blue. IEF was carried out at $60 \mathrm{kVh}$ followed by SDS-PAGE on gels $(12.52 \% \mathrm{~T}, 0.97 \% \mathrm{C})$ that were then stained with Coomassie Blue. 


\subsection{Protein Identification by Mass Spectrometry (MS)}

Selected spots were excised and treated with successive washes of ammonium bicarbonate $\left(\mathrm{NH}_{4} \mathrm{HCO}_{3}\right)$ plus acetonitrile $(\mathrm{ACN})$ in order to remove Coomassie Blue. To dehydrate the gel spots, they were incubated in $100 \% \mathrm{ACN}$ then the ACN was completely removed.

For in-gel digestion at $37{ }^{\circ} \mathrm{C}$ overnight, $15 \mu \mathrm{L}$ of $10 \mathrm{ng} / \mu \mathrm{L}$ trypsin (V5111; Promega, Madison, WI, USA) in $25 \mathrm{mM} \mathrm{NH}_{4} \mathrm{HCO}_{3}, 15 \mu \mathrm{L}$ of $10 \mathrm{ng} / \mu \mathrm{L}$ chymotrypsin (C6423, Sigma, St. Louis, MO, USA) in $50 \mathrm{mM} \mathrm{NH} \mathrm{HCO}_{3}$, and $10 \mathrm{mM} \mathrm{CaCl}$ were added to the dry gel. Gel pieces were centrifuged and $10 \mu \mathrm{L} \mathrm{ACN}$ was added to extract the peptides. The mixture was sonicated for $5 \mathrm{~min}$ and centrifuged at $5000 \mathrm{~g}$ for $5 \mathrm{~min}$. For matrix-assisted laser-desorption/ionization time of flight mass spectrometry (MALDI-TOF-MS), $1 \mu \mathrm{L}$ of supernatant was loaded directly onto the MALDI target. One microliter of the matrix solution $(5 \mathrm{mg} / \mathrm{mL} \alpha$-cyano-4-hydroxycinnamic acid in $50 \%$ ACN/0.1\% trifluoroacetic acid) was added immediately and the mixture was left to dry at room temperature.

Peptide mass fingerprinting was performed using a Voyager DE-Pro model of MALDI-TOF mass spectrometer (Perspective BioSystems, Farmingham, MA, USA) used in positive-ion reflector mode. External calibration was performed with a standard peptide solution (ProteomiX 3, LaserBio Labs, Sophia-Antipolis, France). Internal calibration was performed using peptides resulting from auto-digestion of trypsin. Mono-isotopic peptide masses were obtained using MALDI-TOF-MS analysis and were searched against the NCBI non-redundant protein sequence database using the MASCOT tool (Matrix Science). Matches to protein sequences from the Viridiplantae taxon were considered acceptable if a significant score was obtained from MASCOT, which rates scores as significant if they are above the $95 \%$ significance threshold $(p<0.05)$. Protein scores greater than 70 were considered significant.

The $M_{\mathrm{r}}$ of intact HMW-GS 1 and 1.1 was measured according to Liu et al. [41].

\subsection{Analysis of the HMW-GS 1Ax1.1 Coding Sequence}

DNA extraction was carried out according to Stein et al. [42]. PCR was performed in a reaction volume of $25 \mu \mathrm{L}$ using $25 \mathrm{ng}$ of genomic DNA, 1 U Taq DNA polymerase in 1× PCR buffer, $200 \mu \mathrm{M}$ dNTP mix and 10 pmol of each primer. Primers for the HMW-GS 1 Ax1.1 coding sequence were: forward 5'-CGAGATGACTAAGCGGTTGGTT-3' and reverse 5'-GAGTTCTATCACTGGCTGGCCA-3'. The PCR reaction started with an initial cycle at $94{ }^{\circ} \mathrm{C}$ for 4 min, followed by 35 cycles of $94{ }^{\circ} \mathrm{C}$ for $1 \mathrm{~min}, 65^{\circ} \mathrm{C}$ for $1 \mathrm{~min}$ and $72{ }^{\circ} \mathrm{C}$ for $2 \mathrm{~min}$, then a final extension at $72{ }^{\circ} \mathrm{C}$ for $5 \mathrm{~min}$.

PCR amplification products were analyzed by agarose gel electrophoresis in $1 \%$ agarose and stained with ethidium bromide. The $1 \mathrm{Ax} 1.1$ sequence obtained was deposited in GenBank ${ }^{\circledR}$ under the accession number JN172932.1. Open reading frame (ORF) detection was carried out with the ORF Finder software from NCBI. The 1Ax1.1 sequences alignment was performed with ClustalW2 tool from EBI. 


\section{Conclusions}

The study provides a better understanding of the genetic and molecular basis of the unique HMW-GS 1.1. Although no significant differences were found in some technological tests, this subunit potentially influences polymer size and hence bread-making quality. The 'Barbela' lines, including those bearing the 1.1 subunit, have already been associated with good biscuit quality [24].

Reporting a new x-type allele at the Glu-Al locus means that the genetic variability available for selection is increased. It is expected that through advanced technologies, such as proteomics, the identification of novel or unusual alleles from the large wheat gene pool will provide tools for breeders to further improve dough properties and gluten quality. Proteomics is a powerful tool to elucidate gluten protein expression, diversity and interactions. The resulting knowledge will allow a better conservation of wheat genetic resources. In the future, it would be interesting to extend our knowledge of this novel HMW-GS allele at the molecular level, its heritability (extensibility is a character with low heritability) and its role in determining end use value.

\section{Acknowledgments}

We thank Anne Marie Lugand for her valuable assistance in the technological evaluation of grain. Miguel Ribeiro has a PhD fellowship granted by FCT-Fundação para a Ciência e a Tecnologia and ESF-European Social Fund (SFRH/BD/82334/2011).

\section{References}

1. Osborne, T.B. The Proteins of the Wheat Kernel; Carnegie Institution of Washington: Washington, DC, USA, 1907.

2. Lawrence, G.J.; Shepherd, K.W. Variation in glutenin protein subunits of wheat. Aust. J. Biol. Sci. 1980, 33, 221-233.

3. Payne, P.I.; Holt, L.M.; Jackson, E.A.; Law, C.N.; Damania, A.B. Wheat storage proteins: Their genetics and their potential for manipulation by plant breeding. Philos. Trans. R. Soc. Lond. B Biol. Sci. 1984, 304, 359-371.

4. Macritchie, F. Physicochemical Properties of Wheat Proteins in Relation to Functionality. In Advances in Food and Nutrition Research; John, E.K., Ed.; Academic Press: New York, NY, USA, 1992; Volume 36, pp. 1-87.

5. Payne, P.; Holt, L.; Law, C. Structural and genetical studies on the high-molecular-weight subunits of wheat glutenin. Theor. Appl. Genet. 1981, 60, 229-236.

6. Tatham, A.S.; Miflin, B.J.; Shewry, P.R. The beta-turn conformation in wheat gluten proteinsRelationship to gluten elasticity. Cereal Chem. 1985, 62, 405-412.

7. Tatham, A.S.; Drake, A.F.; Shewry, P.R. Conformational studies of synthetic peptides corresponding to the repetitive regions of the High-molecular-weight (Hmw) glutenin subunits of wheat. J. Cereal Sci. 1990, 11, 189-200.

8. Shewry, P.R.; Halford, N.G.; Lafiandra, D. Genetics of wheat gluten proteins. Adv. Genet. 2003, 49, 111-184. 
9. Shewry, P.R.; Tatham, A.S. Disulphide bonds in wheat gluten proteins. J. Cereal Sci. 1997, 25, 207-227.

10. Buonocore, F.; Bertini, L.; Ronchi, C.; Bekes, F.; Caporale, C.; Lafiandra, D.; Gras, P.; Tatham, A.S.; Greenfield, J.A.; Halford, N.G. Expression and functional analysis of Mr 58,000 peptides derived from the repetitive domain of high molecular weight glutenin subunit $1 \mathrm{Dx} 5$. J. Cereal Sci. 1998, 27, 209-215.

11. Anderson, O.D.; Greene, F.C.; Yip, R.E.; Halford, N.G.; Shewry, P.R.; Malpica-Romero, J.-M. Nucleotide sequences of the two high-molecular-weight glutenin genes from the D-genome of a hexaploid bread wheat, Triticum aestivum L. cv Cheyenne. Nucleic Acids Res. 1989, 17, 461-462.

12. Juhász, A.; Tamás, L.; Karsai, I.; Vida, G.; Láng, L.; Bedő, Z. Identification, cloning and characterisation of a HMW-glutenin gene from an old Hungarian wheat variety, Bánkúti 1201. Euphytica 2001, 119, 75-79.

13. Veraverbeke, W.S.; Delcour, J.A. Wheat Protein Composition and Properties of Wheat Glutenin in Relation to Breadmaking Functionality. Crit. Rev. Food Sci. Nutr. 2002, 42, 179-208.

14. Anjum, F.M.; Khan, M.R.; Din, A.; Saeed, M.; Pasha, I.; Arshad, M.U. Wheat gluten: High molecular weight glutenin subunits-Structure, genetics, and relation to dough elasticity. J. Food Sci. 2007, 72, R56-R63.

15. Tilley, K.A.; Benjamin, R.E.; Bagorogoza, K.E.; Okot-Kotber, B.M.; Prakash, O.; Kwen, H. Tyrosine cross-links: Molecular basis of gluten structure and function. J. Agric. Food Chem. 2001, 49, 2627-2632.

16. Shewry, P.R.; Halford, N.G.; Tatham, A.S. High molecular weight subunits of wheat glutenin. J. Cereal Sci. 1992, 15, 105-120.

17. Gianibelli, M.C.; Larroque, O.R.; MacRitchie, F.; Wrigley, C.W. Biochemical, genetic, and molecular characterization of wheat glutenin and its component subunits. Cereal Chem. 2001, 78, 635-646.

18. Payne, P.I.; Lawrence, G.J. Catalogue of alleles for the complex gene loci Glu-A1, Glu-B1 and Glu-D1 which code for high-molecular-weight subunits of glutenin in hexaploid wheat. Cereal Res. Commun. 1983, 11, 29-35.

19. Branlard, G.; Autran, J.-C.; Rousset, M.; Dardevet, M.E.; Koenig, J. Catalogue des sous unités de haut poids moléculaire des gluténines des blés (T. aestivum e T. durum); Rapport AIP 'Qualité des Blés', Ed.; INRA: Paris, France, 1990; p. 60.

20. Shewry, P.R.; Popineau, Y.; Lafiandra, D.; Belton, P. Wheat glutenin subunits and dough elasticity: Findings of the EUROWHEAT project. Trends Food Sci. Technol. 2000, 11, 433-441.

21. Igrejas, G.; Branlard, G.; Carnide, V.; Gateau, I.; Guedes-Pinto, H. Storage protein diversity within the old Portuguese bread wheat Barbela population. J. Genet. Breed. 1997, 51, 167-173.

22. Igrejas, G.; Guedes-Pinto, H.; Carnide, V.; Branlard, G. The high and low molecular weight glutenin subunits and $\omega$-gliadin composition of bread and durum wheats commonly grown in Portugal. Plant Breed. 1999, 118, 297-302.

23. Ribeiro, M.; Carvalho, C.; Carnide, V.; Guedes-Pinto, H.; Igrejas, G. Towards allelic diversity in the storage proteins of old and currently growing tetraploid and hexaploid wheats in Portugal. Genet. Resour. Crop Evol. 2011, 58, 1051-1073. 
24. Igrejas, G.; Guedes-Pinto, H.; Carnide, V.; Clement, J.; Branlard, G. Genetical, biochemical and technological parameters associated with biscuit quality. II. Prediction using storage proteins and quality characteristics in a soft wheat population. J. Cereal Sci. 2002, 36, 187-197.

25. Li, Q.Y.; Yan, Y.M.; Wang, A.L.; An, X.L.; Zhang, Y.Z.; Hsam, S.L. K.; Zeller, F.J. Detection of HMW glutenin subunit variations among 205 cultivated emmer accessions (Triticum turgidum ssp. dicoccum). Plant Breed. 2006, 125, 120-124.

26. Gobaa, S.; Kleijer, G.; Stamp, P. $2 \cdot$, a new high molecular weight glutenin subunit coded by Glu-A1: Its predicted structure and its impact on bread-making quality. Plant Breed. 2007, 126, $1-4$.

27. Payne, P.I.; Nightingale, M.A.; Krattiger, A.F.; Holt, L.M. The relationship between HMW glutenin subunit composition and the bread-making quality of British-grown wheat varieties. J. Sci. Food Agric. 1987, 40, 51-65.

28. Halford, N.G.; Field, J.M.; Blair, H.; Urwin, P.; Moore, K.; Robert, L.; Thompson, R.; Flavell, R.B.; Tatham, A.S.; Shewry, P.R. Analysis of HMW glutenin subunits encoded by chromosome $1 \mathrm{~A}$ of bread wheat (Triticum aestivum L.) indicates quantitative effects on grain quality. Theor. Appl. Genet. 1992, 83, 373-378.

29. Qian, Y.; Preston, K.; Krokhin, O.; Mellish, J.; Ens, W. Characterization of wheat gluten proteins by HPLC and MALDI TOF mass spectrometry. J. Am. Soc. Mass Spectrom. 2008, 19, 1542-1550.

30. Hickman, D.R.; Roepstorff, P.; Shewry, P.R.; Tatham, A.S. Molecular weights of high molecular weight subunits of glutenin determined by mass spectrometry. J. Cereal Sci. 1995, 22, 99-103.

31. Mamone, G.; de Caro, S.; di Luccia, A.; Addeo, F.; Ferranti, P. Proteomic-based analytical approach for the characterization of glutenin subunits in durum wheat. J. Mass Spectrom. 2009, 44, 1709-1723.

32. Don, C.; Mann, G.; Bekes, F.; Hamer, R.J. Linking Glutenin Particle size to HMW Glutenin Subunit Composition. In The Gluten Proteins; The Royal Society of Chemistry: London, UK, 2004; pp. 285-287.

33. Pritchard, P.E. The glutenin fraction (gel-protein) of wheat protein-A new tool in the prediction of baking quality. Aspects Appl. Biol. 1993, 36, 75-84.

34. Weegels, P.L.; vandePijpekamp, A.M.; Graveland, A.; Hamer, R.J.; Schofield, J.D. Depolymerisation and re-polymerisation of wheat glutenin during dough processing. 1. Relationships between glutenin macropolymer content and quality parameters. J. Cereal Sci. 1996, 23, 103-111.

35. Kelfkens, M.; Lichtendonk, W.J. Verbesserungen in der Beurteilung der Backfahigkeit von Weizensorten. Getreide Mehl und Brot 2000, 54, 363-365.

36. D’Ovidio, R.; Anderson, O.D.; Masci, S.; Skerritt, J.; Porceddu, E. Construction of novel wheat High-Mr glutenin subunit gene variability: Modification of the repetitive domain and expression in E. coli. J. Cereal Sci. 1997, 25, 1-8.

37. D’Ovidio, R.; Lafiandra, D.; Porceddu, E. Identification and molecular characterization of a large insertion within the repetitive domain of a high-molecular-weight glutenin subunit gene from hexaploid wheat. TAG Theor. Appl. Genet. 1996, 93, 1048-1053. 
38. He, G.Y.; Jones, H.D.; D’Ovidio, R.; Masci, S.; Chen, M.; West, J.; Butow, B.; Anderson, O.D.; Lazzeri, P.; Fido, R.; Shewry, P.R. Expression of an extended HMW subunit in transgenic wheat and the effect on dough mixing properties. J. Cereal Sci. 2005, 42, 225-231.

39. Singh, N.K.; Shepherd, K.W.; Cornish, G.B. A simplified SDS-PAGE procedure for separating LMW subunits of glutenin. J. Cereal Sci. 1991, 14, 203-208.

40. Dumur, J.; Jahier, J.; Bancel, E.; Lauriere, M.; Bernard, M.; Branlard, G. Proteomic analysis of aneuploid lines in the homeologous group 1 of the hexaploid wheat cultivar Courtot. Proteomics 2004, 4, 2685-2695.

41. Liu, L.; Wang, A.; Appels, R.; Ma, J.; Xia, X.; Lan, P.; He, Z.; Bekes, F.; Yan, Y.; Ma, W. A MALDI-TOF based analysis of high molecular weight glutenin subunits for wheat breeding. J. Cereal Sci. 2009, 50, 295-301.

42. Stein, N.; Herren, G.; Keller, B. A new DNA extraction method for high-throughput marker analysis in a large-genome species such as Triticum aestivum. Plant Breed. 2001, 120, 354-356.

(C) 2013 by the authors; licensee MDPI, Basel, Switzerland. This article is an open access article distributed under the terms and conditions of the Creative Commons Attribution license (http://creativecommons.org/licenses/by/3.0/). 